\title{
Quality Assurance (QA) Tool in Public Health Campaigns: A Look at the 2017 LLIN Replacement Campaign in Nigeria
}

\author{
Jalal-Eddeen Abubakar Saleh ${ }^{*}$, Wondimagegnehu Alemu², Akubue Augustine Uchenna1, \\ Abdullahi Saddiq ${ }^{1}$, Rex Mpazanje ${ }^{1}$, Bala Mohammed Audu ${ }^{3}$ \\ ${ }^{1}$ Communicable and Non-CommunicableDiseases, World Health Organization, Abuja, Nigeria \\ ${ }^{2}$ WR, World Health Organization Country Office, Abuja, Nigeria \\ ${ }^{3}$ National Malaria Elimination Programmes, Abuja, Nigeria \\ Email: *saleh@who.int
}

How to cite this paper: Saleh, J.-E.A., Alemu, W., Uchenna, A.A., Saddiq, A., Mpazanje, R. and Audu, B.M. (2018) Quality Assurance (QA) Tool in Public Health Campaigns: A Look at the 2017 LLIN Replacement Campaign in Nigeria. Open Access Library Journal, 5: e4701.

https://doi.org/10.4236/oalib.1104701

Received: June 1, 2018

Accepted: June 23, 2018

Published: June 26, 2018

Copyright $\odot 2018$ by authors and Open Access Library Inc.

This work is licensed under the Creative Commons Attribution International License (CC BY 4.0).

http://creativecommons.org/licenses/by/4.0/

\begin{abstract}
Background: Although there are various malaria intervention measures, the long-lasting insecticidal nets (LLIN) are considered as the most cost-effective intervention measure for malaria endemic countries. In line with the Global Technical Strategies, and as recommended by the World Health Organization (WHO), the other available malaria preventive measures to achieve malaria control and elimination in especially the malaria endemic countries include the intermittent prophylactic treatment in pregnancy (IPTp), intermittent prophylactic treatment in infants (IPTi), indoor residual spray (IRS), seasonal malaria chemoprophylaxis (SMC), and recently the use of malaria vaccine. This study examines the role of quality assurance (QA) tool as deployed by WHO in the 2017 LLIN replacement campaigns in the states that implemented the campaign in Nigeria-Adamawa, Edo, Imo, Kwara, and Ondo. For the purpose of this study, the QA tool examined four components during the campaign-logistics, strategies, technical, and demand creation. Methods: This is a cross-sectional study using the QA checklist developed and applied by the WHO professional officers intra campaign between April and December 2017. In each of the states, a total of six LGAs were randomly selected using the EPI risk status (AFP performance indicators and the routine immunization coverage). The findings from the QA checklist tool were analyzed using the SPSS version 24 and the results discussed accordingly. Results: The results looked at general and specific issues across the five states. While the general issues are more in Kwara state in comparison to the other four states-logistics (15\%), strategies (12\%), technical (13\%), and demand creation (7\%), the specific issues are almost same across the five states; these specific issues are poor crowd control (12\%), early closure of distribution sites
\end{abstract}


(14\%), mix-up of data at the distribution sites (15\%), poor communication medium between supervisors and teams at the field (11\%), safety concerns by the recipients (10\%), lack of adequate knowledge on the LLIN usage (9\%), inadequate LLIN storage sites (13\%), and inadequate plans for LLIN movement between the distribution points (16\%). In spite this; all the five states had at least $80 \%$ in the area of programme ownership. Conclusion: On the overall, the study further underscores the importance of using quality assurance checklists in public health campaigns as they help ensure that campaigns meet the minimum required standard.

\section{Subject Areas}

Public Health

\section{Keywords}

Malaria, Long-Lasting Insecticidal Nets, Quality Assurance, National Malaria Elimination Programme, Adamawa, Edo, Imo, Kwara, Ondo, Nigeria

\section{Background}

In spite the drop in the global incidence of malaria among the population at risk by 21 percent [1] and a significant global decline in malaria mortality by 62 percent between 2000 and 2015, and by 29 percent between 2010 and 2015, malaria still remains a major public health challenge especially in the sub-Saharan African region. Although Nigeria and the Democratic Republic of Congo (DRC) remain the epicenters of malaria transmission with a contribution of at least 40 percent of the global malaria burden [2], the 2015 Nigeria malaria indicator survey (NMIS) shows a decline in malaria prevalence from $42 \%$ in 2010 to $27 \%$ in 2015 [3] [4] [5].

Although there are various malaria intervention measures, the long-lasting insecticidal nets (LLIN) are considered as the most cost-effective intervention measure for malaria endemic countries; this is further supported by various studies in that regard [6] [7] [8]. In line with the Global Technical Strategies, and as recommended by the World Health Organization (WHO), the other available malaria preventive measures to achieve malaria control and elimination in especially the malaria endemic countries include the intermittent prophylactic treatment in pregnancy (IPTp), intermittent prophylactic treatment in infants (IPTi), indoor residual spray (IRS), seasonal malaria chemoprophylaxis (SMC), and recently the use of malaria vaccine.

Nigeria, in its quest to achieve the universal LLIN coverage, commenced the LLIN replacement campaign in phases in 2017 [9] [13]. The first long lasting insecticidal net (LLIN) mass campaign took place between 2009-2013 [6]. This is in addition to a continuous distribution channel through antenatal care clinics and the routine immunization of infants. In line with the NMSP, the replace- 
ment campaign targets at least $80 \%$ of households to own two or more LLINs and at least $80 \%$ of children under five and pregnant women to use the LLIN by sleeping inside by 2010 with sustained levels [10] [11].

The Nigerian government, in partnership with the RBM partners, successfully distributed \& achieved over 90 percent target distributing at least 57.7 million LLINs during the last campaign [12]. The 2017 replacement campaign currently on-going across the country, is a support from the Global Fund and with technical support from the World Health Organization.

This study examines at the role of quality assurance (QA) tool as deployed by the WHO in the 2017 LLIN replacement campaigns. For the sole purpose of this study, the QA tool critically examined four components during the campaign-logistics, strategies, technical, and demand creation. The campaign rolled out in phases across five states, Adamawa, Edo, Imo, Kwara and Ondo, between April and December 2017 (Figure 1). The WHO provided an oversight and QA functions throughout the campaign to complement the effort of the $\mathrm{Na}$ tional Malaria Elimination Programme (NMEP).

\section{Method}

This is a cross-sectional study using the QA checklist developed and applied by the WHO professional officers during the LLIN replacement campaign that took place between April and December 2017. The QA tool was administered to teams at the distribution points in each of the states that implemented the campaign to gauge the quality of the campaign from the build-up through to the five days distribution period. The three key areas the tool considered are programme ownership, general issues, and specific issues. In each of the states, a total of six LGAs were randomly selected using the EPI risk status (AFP performance indicators and the routine immunization coverage). While the general issues were on logistics, strategy, technical and demand creation, the specific issues centered on closure of distribution points, LLIN storage sites, LLIN movements, message on LLIN usage by the health educators, data handling, communication strategy between the teams and senior supervisors, and crowd control measures in place at the distribution sites. Similarly, some recipients of the LLIN at the exit points were asked questions that relate to LLIN safety concerns. These findings were analyzed using the SPSS version 24.

\section{Result}

Out of the five states that implemented the LLIN campaign, the gaps identified in the four general areas across the states, and as shown in Figure 2, are Adamawa-logistics (5\%), strategies (4\%), technical (4\%), and demand creation (6\%); Edo-logistics (14\%), strategies (9\%), technical (11\%), and demand creation (8\%); Imo-logistics (5\%), strategies (4\%), technical (4\%), and demand creation (6\%); Kwara-logistics (15\%), strategies (12\%), technical (13\%), and demand creation (7\%), and Ondo-logistics (12\%), strategies (8\%), technical (6\%), and demand creation (6\%). 


\section{LLIN campaign states}

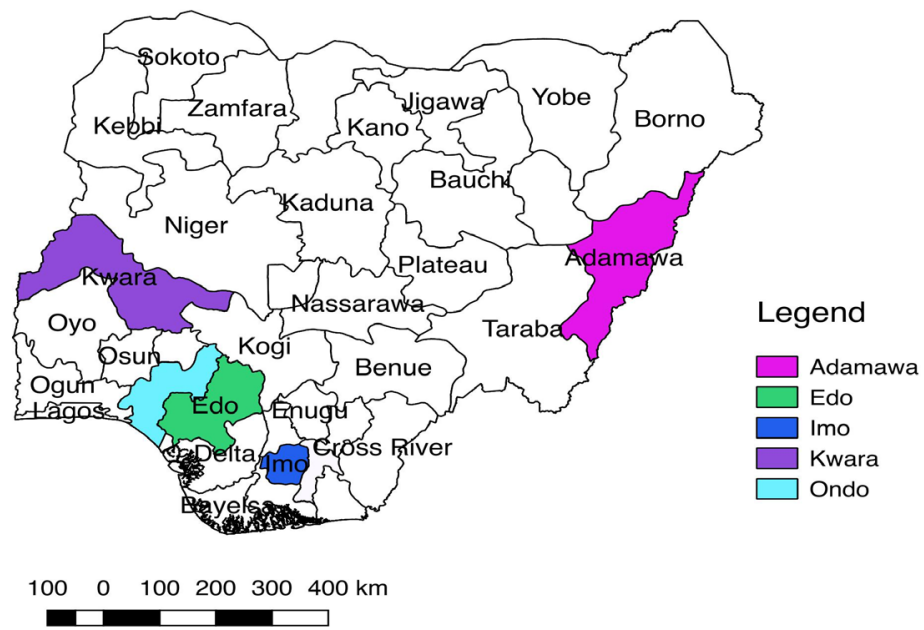

Figure 1. QGIS showing the five implementing states during the LLIN campaign.

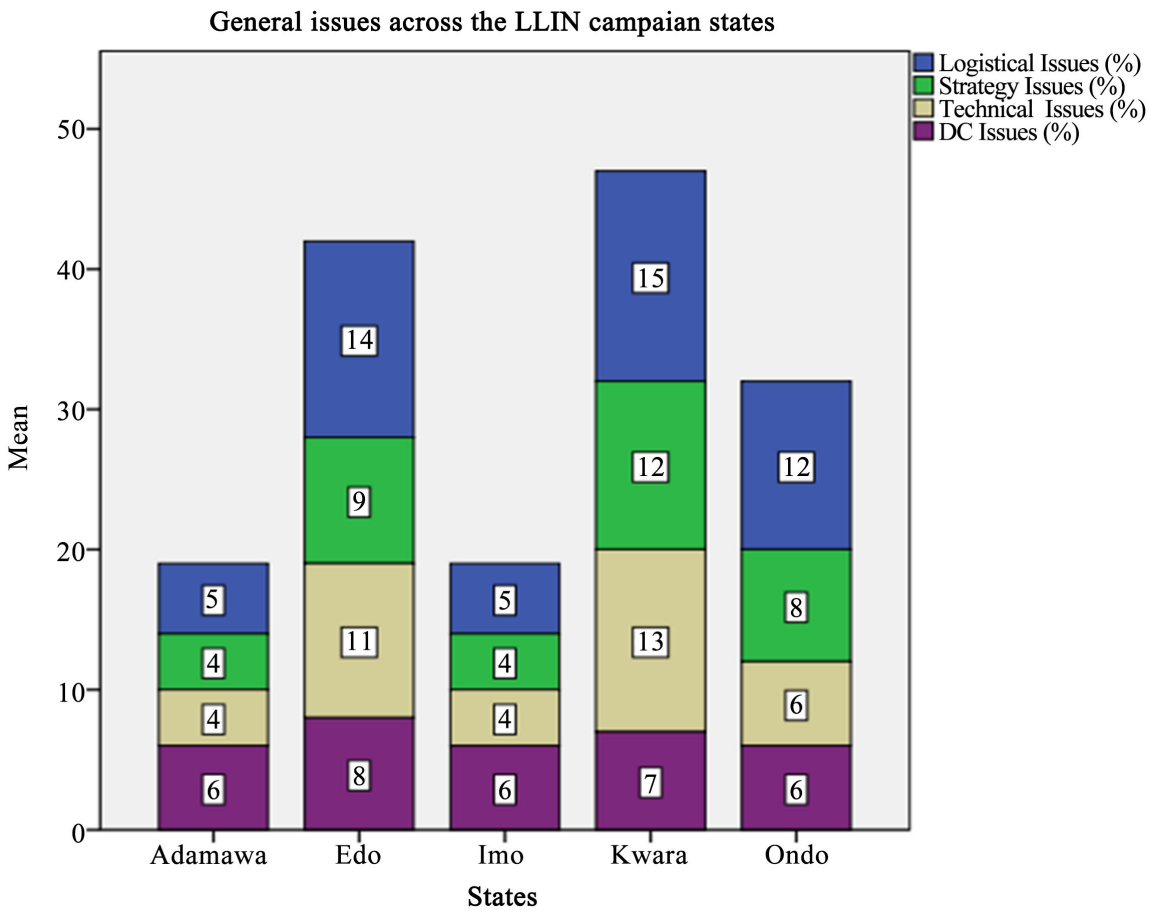

Figure 2. Showing general issues across the LLIN campaign states.

Similarly, and as shown in Figure 3, the specific issues, peculiar to all the states, are inadequate plans for LLIN movement between the distribution points (16\%) appears to top the list across all the states, followed by a mix-up of data at the distribution sites (15\%), early closure of distribution sites (14\%), inadequate LLIN storage sites (13\%), poor crowd control (12\%), poor communication medium between supervisors and teams at the field (11\%), safety concerns by the recipients (10\%), and lack of adequate knowledge on the LLIN usage (9\%). 
Furthermore, and as displayed in Figure 4, the results on programme ownership shows all the states had at least 80 percent.

\section{Discussion}

This in-depth review of the QA checklist has helped immensely in identifying the weak areas that require attention and also help partners to address these areas to avoid their recurrence in subsequent LLIN campaigns in the remaining states.

The results from the QA checklist tool has shown that of the performances from the five implementing states, Edo had higher issues in the areas of logistics (14\%), strategies (9\%), technical (11\%), and demand creation (8\%). However, part of the reasons for this could be due to the fact that Edo was the first state

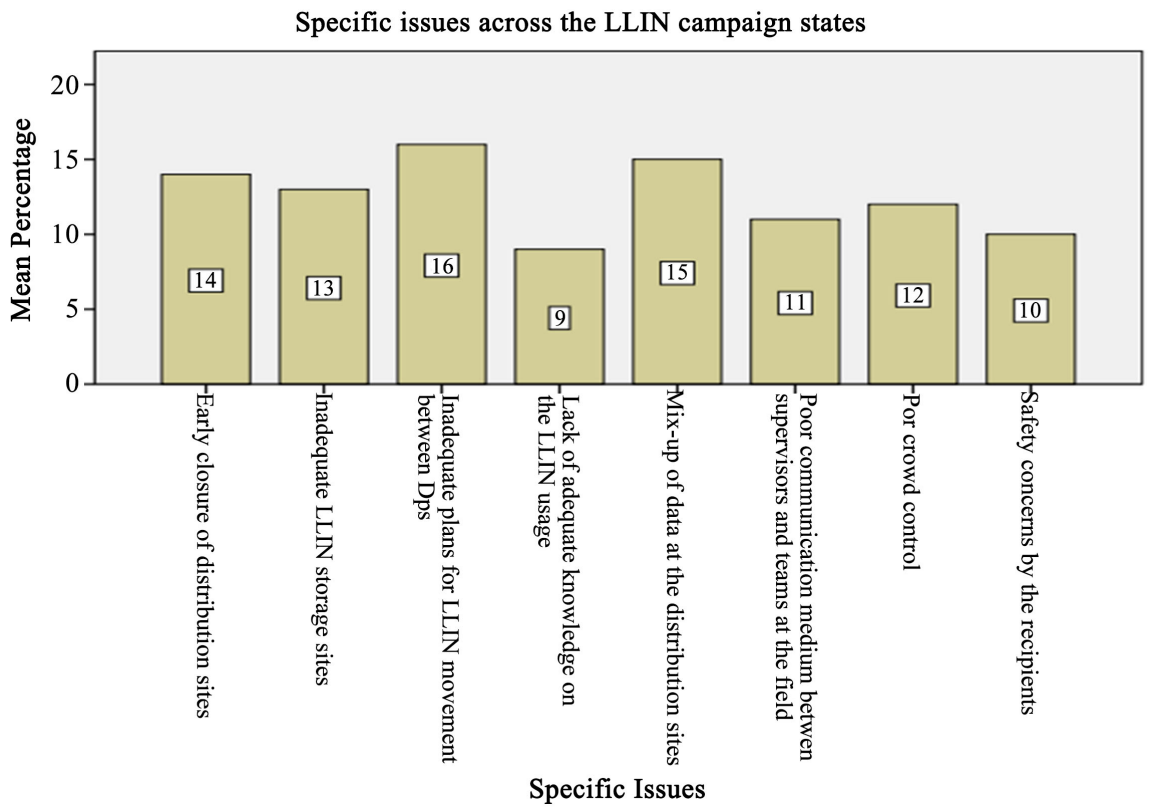

Figure 3. Showing specific issues across the LLIN campaign states.

Programme ownership across the LLIN campaign states
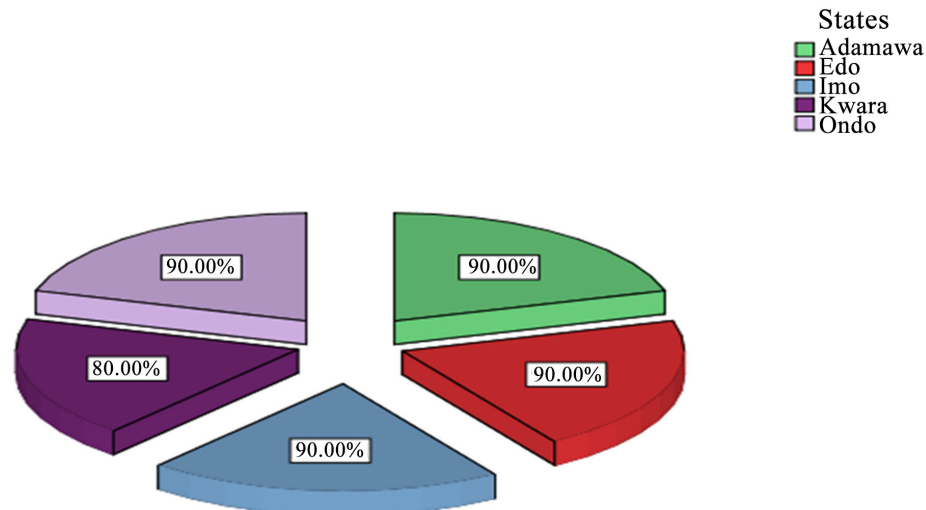

Figure 4. Showing LLIN programme ownership across the LLIN campaign states. 
that implemented the LLIN replacement campaign in 2017 across the country. These gaps, as identified in Edo, were much less in the other implementing states of especially Adamawa and Imo states which had the lowest issues across all the four key indicators. The possible reason for the fewer gaps seen in Adamawa and Imo states could be as a result of intensified planning by the implementers and other key players in the campaign that addressed the weaknesses hitherto identified during implementation in Edo state.

The specific issues, which were almost similar of the same weight across all the five states that had the campaign, has further highlighted the need for the RBM partners to strategize more in addressing these gaps during the subsequent campaigns in the remaining states in 2018 and 2019. These specific issues, which could be considered as the pivot to achieving quality campaign and eventually universal LLIN coverage in Nigeria, include inadequate plans for LLIN movement between the distribution points (16\%), mix-up of data at the distribution sites $(15 \%)$, early closure of distribution sites (14\%), inadequate LLIN storage sites (13\%), poor crowd control (12\%), poor communication medium between supervisors and teams at the field (11\%), safety concerns by the recipients $(10 \%)$, and lack of adequate knowledge on the LLIN usage (9\%).

The inadequate plans for LLIN movement between the distribution points (DP) and early closure of distribution sites could explain the stock out recorded at some of the distribution points. These issues together with poor crowd control results in eligible persons leaving the designated DPs without getting the LLINs, a situation often regarded as missed opportunities. The mix-up of data at some distribution sites results in poor accountability and raising questions about the quality of the personnel engaged in the campaign. Similarly, the issues with inadequate LLIN storage sites often result in pilfering of the nets negatively impacting on the campaign.

Furthermore, teams working at the field that run into problems and wanted clarity on certain issues from their superiors often had difficulties linking up with the supervisors due to poor communication medium thus leaving them to act on their own. This problem is not only limited to one state but cuts across all the five states. Equally, there are safety concerns by the LLIN recipients and the lack of adequate knowledge on LLIN usage by the beneficiaries. This highlights the poor knowledge of the health educators on the key messages to share with the clients at the DP and also the problems of demand creation through the use of available media outlets. The result has highlighted good political commitment across the states as deputy governors and or state commissioners for health actively participated in the process and are available at the flag-off events.

\section{Conclusion}

Overall, this study underscores the fact that quality assurance tool is an important requirement to achieving a successful public health campaign. These tools, when correctly, purposefully, and timely applied would help campaign imple- 
menters and partners to ensure that gaps are identified and addressed early enough in the campaign so as to meet the minimum required standards. Furthermore, the need for partners and stakeholders to work together and assign responsibilities to officers based on the areas of their competencies should be emphasized. There is need for the QA checklist deployed by the WHO at different stages of the LLIN replacement campaign across the five states to be scaled up for use in campaign across the remaining states in 2018 and 2019 so that the country would achieve a quality campaign.

\section{Acknowledgements}

The QA tool deployed by the World Health Organization during the LLIN campaign is part of the Technical Assistance given to the national malaria elimination programme in Nigeria.

We declare that there is no any potential conflict of interest or royalty associated with the research.

We declare that there is no funding received from any source for this research.

\section{References}

[1] World Health Organization (2016) WHO Global Malaria Programme, World Malaria Report 2016. WHO Document Production Service, Geneva.

[2] World Health Organization (2014) WHO Global Malaria Programme, World Malaria Report 2014. WHO Document Production Service, Geneva.

[3] NMEP, NPopC, NBS, and ICF International (2016) Nigeria Malaria Indicator Survey 2015. NMEP, NPopC, and ICF International, Abuja, Nigeria, and Rockville, Maryland, USA.

[4] Saleh, J.-E.A., Wondimagegnehu, A., Mpazanje, R., et al. (2017) Investigation of a Suspected Malaria Outbreak in Sokoto State, Nigeria, 2016. Open Access Library Journal, 4, e4246. https://doi.org/10.4236/oalib.1104246

[5] Saleh, J.-E.A., Saddiq, A. and Uchenna, A.A. (2018) LLIN Ownership, Utilization, and Malaria Prevalence: An Outlook at the 2015 Nigeria Malaria Indicator Survey. Open Access Library Journal, 5, e4280. https://doi.org/10.4236/oalib.1104280

[6] Ntuku, H.M., Ruckstuhl, L., Julo-Réminiac, J.-E., et al. (2017) Long-Lasting Insecticidal Net (LLIN) Ownership, Use and Cost of Implementation after a Mass Distribution Campaign in Kasaï Occidental Province, Democratic Republic of Congo. Malaria Journal, 16, 22. https://doi.org/10.1186/s12936-016-1671-1

[7] Kulkarni, M.A., Eng, J.V., Rachelle, E., Desrochers, R.E., et al. (2010) Contribution of Integrated Campaign Distribution of Long-Lasting Insecticidal Nets to Coverage of Target Groups and Total Populations in Malaria-Endemic Areas in Madagascar. The American Journal of Tropical Medicine and Hygiene, 82, 420-425. https://doi.org/10.4269/ajtmh.2010.09-0597

[8] FMoH Nigeria (2009) National Malaria Strategic Plan 2009-2013: A Road Map for Malaria Control in Nigeria. NMCP, Abuja.

[9] Saleh, J.-E.A., Uchenna, A.A., Saddiq, Dr.A., Wondimagegnehu, A., Mpazanje, R. and Audu, Dr.B.M. (2018) Lots Quality Assurance Survey (LQAS) as a Strategy to Achieving Quality LLIN Campaigns: The Nigerian Experience. Open Access Library Journal, 5, e4484. https://doi.org/10.4236/oalib.1104484 
[10] The Roll Back Malaria Partnership (2008) The Global Malaria Action Plan-for a Free Malaria. UNHCR. http://www.unhcr.org/4afac5629.pdf

[11] FMoH Nigeria (2014) National Malaria Strategic Plan 2014-2020: A Road Map for Malaria Control in Nigeria. NMCP, Abuja.

[12] Robertson, S.E., Anker, M., Roisin, A.J., et al. (1997) The Lot Quality Technique: A Global Review of Applications in the Assessment of Health Services and Disease Surveillance. World Health Statistics Quarterly, 50, 199-209.

[13] Uchenna, A.A., Saleh, J.-E.A., Saddiq, A., Wondimagegnehu, A. and Mpazanje, R. (2018) The Role of Dashboards in Long Lasting Insecticidal Nets (LLIN) Campaigns: A Pilot Report from Nigeria. (In print) 